\title{
Optimization design and development of UHF omnidirectional wideband antenna for PD detection in substation
}

\author{
Zhen-hua Xing ${ }^{1, *}$, Zhen Wang ${ }^{1}$, Hang Xu${ }^{1}$, Miao Xu${ }^{1}$, Xiao-lin Mou ${ }^{1}$, Lu-xiao Wu ${ }^{1}$, Kun Yang ${ }^{1}$, Zhao Liu ${ }^{1}$ \\ ${ }^{1}$ State Grid Taian Power Supply Company, Taian, Shandong, 271000, China
}

\begin{abstract}
In order to meet the requirements of partial discharge (PD) detection of electrical equipment in substation station area, the ultra-high frequency (UHF) antenna with broadband, high gain and horizontal plane (H-plane) omnidirectional is studied and designed as a PD sensor. By analyzing the characteristic impedance and structural characteristics of the biconic antenna, it is determined to be an UHF omnidirectional wideband antenna for detecting PD. The influence of structural parameters such as half-cone angle and vertebral body height on the antenna performance is simulated. At last, a double cone antenna is made and its bandwidth, directional pattern, gain and equivalent height are tested.
\end{abstract}

\section{Introduction}

Substation is an important link of electric energy transmission between power plant and users, and the failure of substation equipment seriously threatens the safe and reliable operation of power system [1-3]. The experience of power network operation shows that the main form of insulation failure in the early stage is PD, which is not only the main cause of insulation deterioration, but also the characteristic quantity of the status of insulation. Therefore, PD testing is generally regarded as an important means to diagnose the insulation condition of equipment. When PD occurs in power equipment, the radiated electromagnetic wave signal can leak into the substation space through the non-metallic shielding parts, and the electric field intensity is approximately inversely proportional to the propagation distance, which can be detected at a distance by the highsensitivity UHF antenna. Therefore, the non-contact UHF is the best method for PD detection in substations [4-7].

In order to realize the omnidirectional PD detection and localization of substation, the detection antenna is generally required to have uniform omnidirectional and wide band performance. Moore et al. developed the omnidirectional discone antenna, which is composed of a vertebral body and a grounded disc, and the detection frequency band is $0.1-1.0 \mathrm{GHz}$. Ye et al. adopted a spherical structure loaded on the upper part of the vertebral body and connected the vertebral body and disk with metal stub columns to enhance the mechanical properties of the antenna [8]. Liu et al. optimized the size of the discone antenna through the simulation of return loss and gain, and developed the discrete processing of the disc cone antenna, but the size of the antenna was too large and inconvenient to carry [9]. Based on the theory of broadband monopole antenna, Ye et al. developed a substation local detection antenna with an elliptical monopole patch structure [10]. The detection frequency band is $0.3-2.0 \mathrm{GHz}$, and it has advantages of omnidirectional, broadband and low standing wave ratio. Omnidirectional antenna has the advantage of omnidirectional detection, but its gain is generally low, about OdBi. Zhou et al. developed Vivaldi antenna for directional detection, and its maximum gain can reach 7.9dBi [11]. Directional antenna has the advantages of high detection sensitivity and excellent anti-noise, but requires rotating antenna array to achieve omnidirectional local detection, which reduces the efficiency of field inspection.

In the past, PD detection system is usually only aimed at a single electrical equipment, and the antenna used is not omnidirectional. This kind of antenna could not meet the requirement of $\mathrm{PD}$ detection for electrical equipment in substation. The UHF omni-directional broadband antenna developed in this paper has the characteristics of wide working frequency band, omni-directional and high detection sensitivity in the horizontal plane, which can effectively detect and locate the PD of electrical equipment in substations.

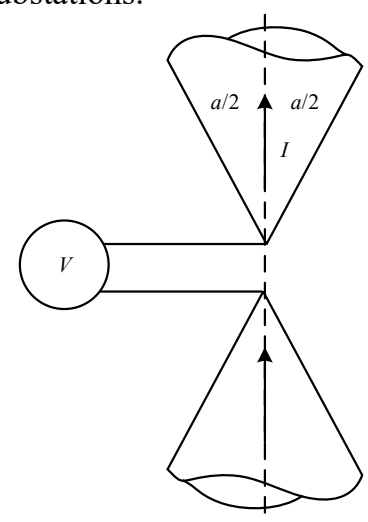

Figure 1. Infinite length biconical antenna 


\section{Analysis and selection of antenna sensor}

\subsection{Design requirements of antenna sensor}

In the design of UHF sensor, the measurement indexes need to pay attention to are bandwidth, pattern, gain and equivalent height. Standing wave ratio is used to characterize the power loss caused by antenna reflection. When the standing wave ratio is less than 2, the antenna has good matching characteristics. $S_{11}$ represents the input return loss of the antenna, representing the ratio of reflected power and incident power at the antenna junction. The smaller the $S_{11}$ parameter is, the better the matching characteristics of the antenna are. When the standing wave ratio is 2 , the corresponding $S_{11}$ parameter is $-9.54 \mathrm{~dB}$. Therefore, in antenna design, the frequency range where the antenna $S_{11}$ parameter is less than $-10 \mathrm{~dB}$ also corresponds to the impedance bandwidth of the antenna. In engineering, the direction diagram of the antenna is commonly used to directly represent the direction of the antenna. Direction diagram is the distribution of radiation characteristics (directivity coefficient or gain, etc.) of the antenna far field in space expressed by graphics, which can be divided into E-plane (vertical plane) direction diagram, H-plane direction diagram and threedimensional direction diagram, etc. The gain of the antenna represents the ability of the antenna to concentrate the input power to the main direction. Under the condition of the same signal strength, the larger the gain of the antenna, the farther the detection distance will be. The equivalent height, also known as the antenna sensitivity, characterizes the antenna's ability to convert the received electromagnetic energy into a voltage signal. In general, the wider the working bandwidth of the antenna is, the greater the equivalent height of the antenna is. For the same incident electric field, the higher the detection sensitivity of the antenna is, and the farther the detection range is.

Antenna sensor should be able to receive electromagnetic wave signals radiated into the air due to corona discharge of electrical equipment from a certain distance $[12,13]$.To facilitate the movement of the antenna array in the substation with limited space, the antenna design must meet the following conditions: the directional pattern on H-plane is omnidirectional, that is, the directional pattern of the antenna on H-plan is circular [14].In the detection of PD in substations, the antenna is required to be able to effectively receive UHF signals of PD from all horizontal directions and have a large impedance bandwidth [15].It is generally believed that the frequency band of PD UHF signal is $0.3-3 \mathrm{GHz}[16]$. In order to extract more PD signal information, the impedance bandwidth of the antenna is required to cover the frequency band of $0.3-3 \mathrm{GHz}$ as far as possible, with a high sensitivity (equivalent height). The size of the antenna is as small as possible, the structure is as simple as possible, the weight is relatively light, easy to transport and install.

\subsection{Theoretical analysis of bicone antenna characteristics}

As mentioned above, substation detection antennas are required to be omnidirectional, and the more uniform the omnidirectional antennas are, the more similar the antennas receive signals. The bicone antenna has a strict axisymmetric structure, and its direction pattern is completely symmetrical around the $Z$ axis, and its gain is the largest in the horizontal direction, so it is suitable for PD detection in substations. In order to facilitate the movement of the antenna array, the distance between the antennas is generally small, and the antenna size should not be too large. With smaller diameter, the bicone antenna is more suitable for the detection and positioning of substation. Therefore, the biconical antenna is selected as the UHF sensor.

An ideal biconical antenna consists of two symmetric infinitely long vertebrae with cone angle $\alpha$, as shown in figure 1 . The two vertebrae are coaxial, and the feeding point of the antenna is at the gap between the vertebrae. When the biconical antenna is used as a receiving antenna, the electromagnetic wave in space causes the antenna to generate induced current $I$ on the surface, thus forming voltage $V$ between the vertebral bodies. For the infinitely long biconical antenna, the input impedance is equal to the characteristic impedance, and the performance of the antenna is independent of the frequency. Therefore, the biconical antenna has typical broadband characteristics, and the antenna sensitivity is relatively high. And because its structure is strictly axisymmetric structure, it also has good omnidirectional characteristics.

\section{Simulation design of bicone antenna}

\subsection{Simulation model of biconical antenna}

A typical biconical antenna of finite length is shown in figure 2 (a). The conical structure is symmetrical from top to bottom. The radius of the bottom of the upper vertebral body is $r_{1}$, the radius of the top is $r_{2}$, the height of the vertebral body is $h$, and the distance of the upper and lower vertebral bodies is $d$. The half-cone angle of the vertebral body is expressed in Equation (1).

$$
\frac{\alpha}{2}=\tan ^{-1}\left(\frac{r_{2}-r_{1}}{h}\right)
$$

In order to design a biconical antenna which can meet the requirement of $\mathrm{PD}$ detection in substation, it is necessary to simulate the influence of the variation of antenna structure parameters on the antenna performance. The bicone antenna itself has a very good omnidirectional and high sensitivity. Therefore, the goal of simulation is to increase the impedance bandwidth of the antenna in the range of $0.3-3 \mathrm{GHz}$ to ensure that the antenna can effectively receive all frequency components of the PD UHF signal. At the same time, the volume of the antenna should also be considered in the simulation, and the volume should not be too large. In order to analyze the influence of the change of structural parameters on the 
impedance performance of the biconical antenna, the simulation software of UHF electromagnetic wave was used to model and simulate the biconical antenna. The simulation model is shown in figure 2 (b). In figure 2 (b), the upper and lower vertebral bodies of the biconical antenna model are symmetrical in structure, and both of

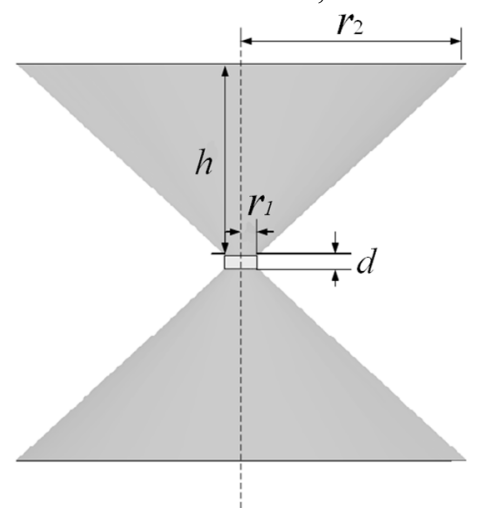

(a) Typical structure of a biconical antenna of finite length them are ideal electrical conductor materials. Gaps between vertebral body for the antenna's feed point, with $50 \Omega$ coaxial wire feeder. The frequency range of simulation is $0.3-3 \mathrm{GHz}$, which corresponds to the frequency range of UHF signal of PD of electrical equipment.

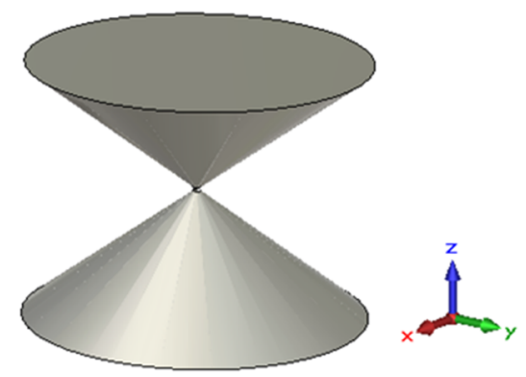

(b) Biconical antenna simulation model

Figure 2. Typical structure diagram and simulation model of the double-cone antenna

\subsection{Influence of half cone angle on the performance of bicone antenna}

Equation (2) gives the relation between the input impedance $Z_{\text {in }}$ of the biconical antenna and the cone angle $\alpha$. It can be seen that the input impedance of the biconical antenna of infinite length is determined by the cone angle $\alpha$. For a biconical antenna of finite length, although each structural parameter has an effect on the input impedance, the most important factor is the half-cone angle of the vertebral body. The height of the vertebral body was set as

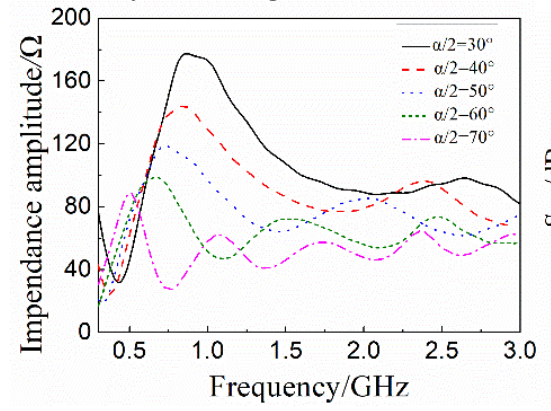

(a) Impedance amplitude

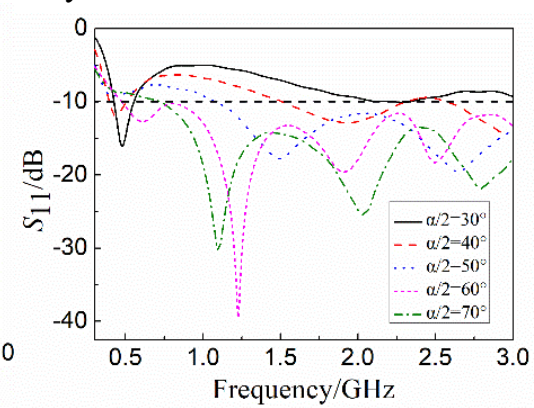

(b)parameter S11 $h=8 \mathrm{~cm}$, the bottom radius of the upper vertebral body was set as $r_{1}=0.4 \mathrm{~cm}$, and the spacing of the vertebral body was set as $d=0.4 \mathrm{~cm}$. The half-cone angles were set as $30^{\circ}, 40^{\circ}$, $50^{\circ}, 60^{\circ}$ and $70^{\circ}$, respectively, and the corresponding top radius $r_{2}$ of the upper vertebral body was $5.0 \mathrm{~cm}, 7.1 \mathrm{~cm}$, $9.9 \mathrm{~cm}, 14.3 \mathrm{~cm}$ and $22.3 \mathrm{~cm}$, respectively. The simulation results are shown in figure 3 .

$$
Z_{\text {in }}=120\left[\cot \left(\frac{\alpha}{4}\right)\right]
$$

Figure 3. Influence of half cone Angle on antenna impedance performance

figure 3 (a) shows the curve of the amplitude of the input impedance of the bicone antenna changing with the half-cone angle. For a biconical antenna of finite length, it can be seen that the input impedance of the antenna will vary with the frequency and is not a fixed value. The amplitude of the antenna input impedance varies greatly in the range of $0.3-3 \mathrm{GHz}$, but less in the high frequency range. When the half-cone angle increases, the amplitude of the antenna input impedance gradually decreases, which is consistent with Equation (2). figure 3 (b) and (c) show the curves of the $S_{11}$ parameters and the standingwave ratio of the biconical antenna changing with the halfcone angle. It can be seen that the half-cone angle has a great influence on the upper and lower frequencies of the antenna impedance bandwidth. In the frequency range of
$0.3-3 \mathrm{GHz}$, when the half-cone angle is greater than or equal to $40^{\circ}$, the $S_{11}$ parameter of the bicone antenna is basically less than $-10 \mathrm{~dB}$ in the high frequency band, and the standing-wave ratio is basically less than 2 in the high frequency band. It shows that when the half-cone angle of the biconical antenna is greater than or equal to $40^{\circ}$, the upper limit frequency of the impedance bandwidth is basically greater than $3 \mathrm{GHz}$. When the half-cone angle increases, the lower frequency decreases and the impedance bandwidth of the antenna increases.

In the design process of the bicone antenna, in order to increase the impedance bandwidth, the half-cone angle should be increased as much as possible. However, when the height of the antenna is constant, the increase of the half-cone angle will also lead to the increase of the radius 
of the upper end of the antenna vertebra, which will increase the volume and weight of the antenna and make it inconvenient to carry and move. After comprehensive consideration, it is determined that the antenna cone angle should be between $50^{\circ}$ and $60^{\circ}$, which can ensure that the antenna volume will not be too large, and the impedance bandwidth is relatively large, meeting the requirements of PD detection of substation.

\subsection{Influence of vertebral height on the performance of the bicone antenna}

In order to study the influence of the height of the upper vertebral body on the antenna impedance bandwidth, the bottom radius of the upper vertebral body was set as $r_{1}=0.4 \mathrm{~cm}$, the spacing of the upper vertebral body $d=0.4 \mathrm{~cm}$, the half-cone Angle $\alpha / 2=60^{\circ}$, the height of the upper vertebral body h was set as $4 \mathrm{~cm}, 6 \mathrm{~cm}, 8 \mathrm{~cm}, 10 \mathrm{~cm}$ and $12 \mathrm{~cm}$ respectively, and the corresponding top radius of the upper vertebral body $r_{2}$ was set as $7.3 \mathrm{~cm}, 10.8 \mathrm{~cm}$, $14.3 \mathrm{~cm}, 17.7 \mathrm{~cm}$ and $21.08 \mathrm{~cm}$ respectively. The simulation results are shown in figure 4.

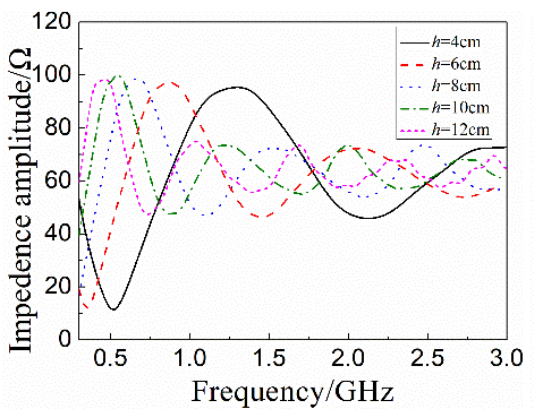

(a) Impedance amplitude

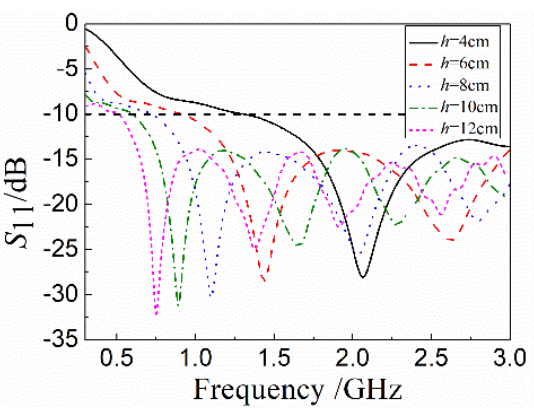

(b)parameter S11

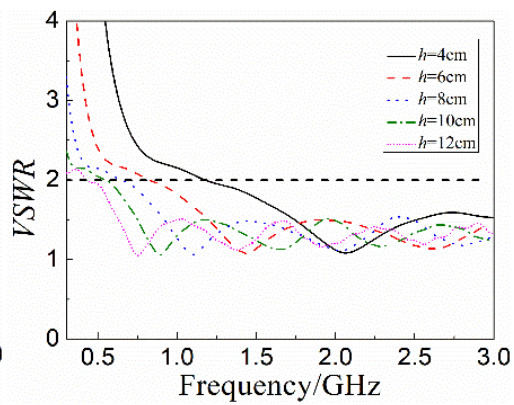

(c) Standing wave ratio

Figure 4. Influence of vertebral body height on antenna impedance performance

figure 4 (a) shows the variation of input impedance amplitude of biconical antenna with vertebral height. The higher the vertebral body height is, the lower the frequency point corresponding to the first peak of input impedance is. The influence of vertebral height on the input impedance is small in the high frequency band, and the input impedance of biconical antenna is gradually stable in the high frequency band. According to formula (2), when the half cone angle is $60^{\circ}$, the impedance of the infinite biconical antenna is $66 \Omega$. It can be seen that the amplitude of input impedance of antenna with different vertebral body height also changes around $66 \Omega$ with the increase of frequency, which also shows that the influence of half cone angle on input impedance is greater than that of vertebral body height.

figure 4 (b) and (c) show the curves of $S_{11}$ parameters and standing wave ratio of biconical antenna varying with vertebral height. It can be seen that the height of vertebral body mainly affects the lower limit frequency of antenna impedance bandwidth. With the increase of vertebral height, the lower limit frequency of antenna impedance bandwidth will decrease, and the impedance bandwidth of antenna in the range of $0.3-3 \mathrm{GHz}$ will increase. Similarly, in the process of antenna design, we also need to consider the problem of antenna volume increase caused by the increase of vertebral height. After comprehensive consideration, the height of antenna vertebral body was determined as $8 \mathrm{~cm}$.

According to the simulation results, increasing the half cone angle and vertebral body height of the biconical antenna can effectively reduce the lower limit frequency of the antenna, thus increasing the impedance bandwidth of the antenna in the range of $0.3-3 \mathrm{GHz}$, but also increasing the volume of the antenna. Considering that the antenna will move in the substation during the experiment, the size of the antenna is determined as follows: the vertebral body height is $8 \mathrm{~cm}$, the radius of the top of the upper vertebral body is $10 \mathrm{~cm}$, the radius of the bottom of the upper vertebral body is $0.2 \mathrm{~cm}$, the cone angle is $52.5^{\circ}$ and the distance between the vertebral bodies is $0.2 \mathrm{~cm}$.

\section{Development and performance test of bicone antenna}

\subsection{Development of bicone antenna}

According to the size of biconical antenna determined by simulation optimization, the biconical antenna is made, and the structure diagram is shown in figure 5. The antenna comprises a shell and upper and lower vertebral bodies in the shell, and the cone angles of the two vertebral bodies are relatively connected together. The vertebral body material of the antenna is aluminum alloy, and the shell material is glass fiber. The shell is mainly used to fix the vertebral body and protect the antenna, which will not affect the receiving performance of the antenna. The two cones of the antenna are connected together by a special $\mathrm{N}$-shaped connector. The $\mathrm{N}$-shaped connector consists of a central guide rod, a polytetrafluoroethylene dielectric layer and an outer shielding connector with an impedance of $50 \Omega$; the central guide rod is threaded through the middle hole of the antenna upper vertebral body and fixed with the upper vertebral body by nuts; the outer shielding connector is threaded and connected with the antenna lower vertebral body and the feed coaxial line. 


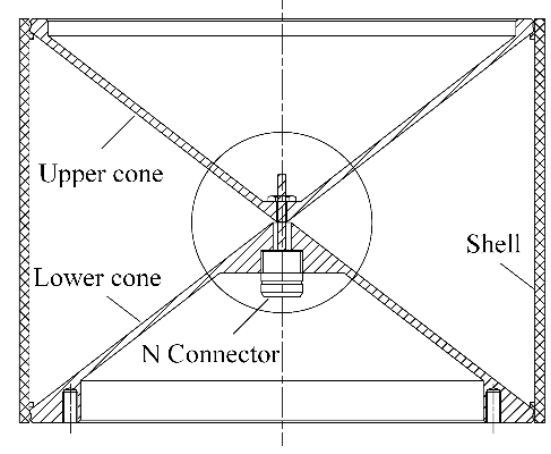

Figure 5. Structure diagram of double-cone antenna

\subsection{Performance test of bicone antenna}

Firstly, the standing wave ratio of the biconical antenna was tested by using a vector network analyzer. The result is shown in figure 6. It can be seen that the measured standing-wave ratio is close to the simulation results, indicating that the antenna performance has reached the expectation after optimization. The standing-wave ratio of the antenna is generally less than 2 in the range of 0.3 $2.5 \mathrm{GHz}$. At the frequency of $0.8-1 \mathrm{GHz}$, the standing wave exceeds 2 , and the maximum value is about 2.35 , which is in good agreement with the simulation results. When the standing-wave ratio is 2.35 , the energy transmission efficiency of the antenna is about $83.8 \%$, which is reduced by $5 \%$ compared with $88.9 \%$ when the standing-wave ratio is 2 . The impedance matching characteristics of the

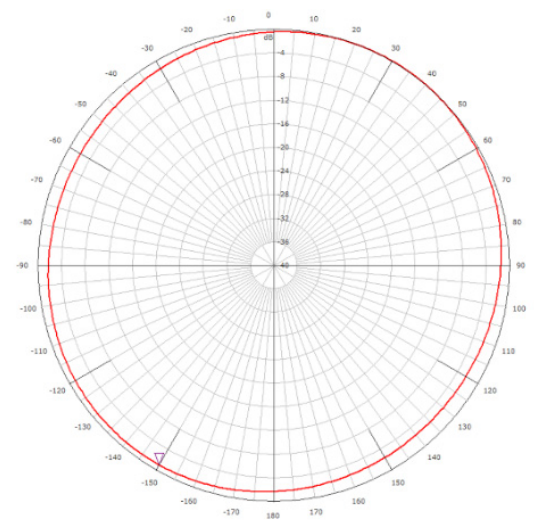

(a) $400 \mathrm{MHz}$

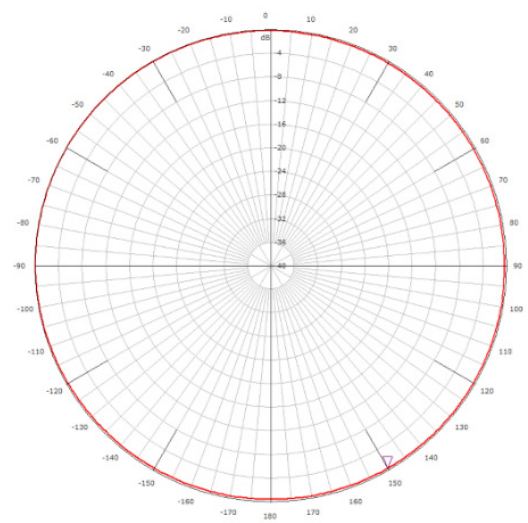

(c) $1200 \mathrm{MHz}$ antenna are also good. The test results show that the bicone antenna has good impedance matching performance in the frequency band of $0.3-2.5 \mathrm{GHz}$.

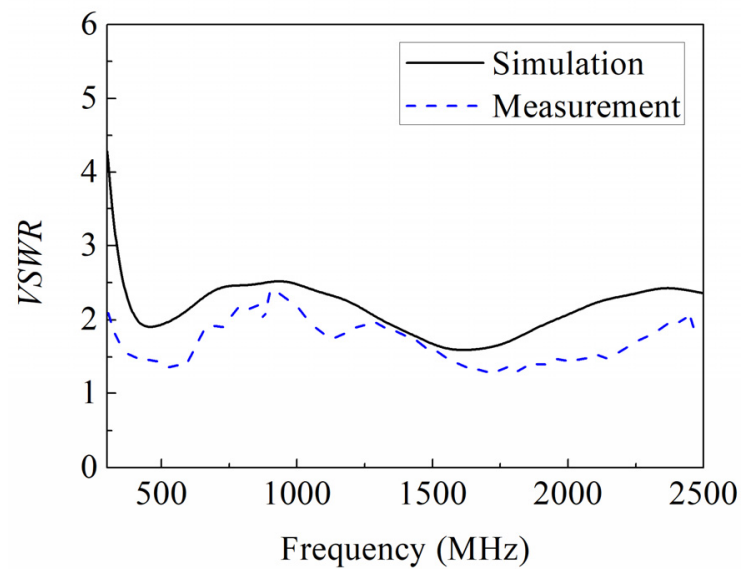

Figure 6. Standing-wave ratio measurement results of the double-cone antenna

figure 7 shows the H-plane pattern given by the thirdparty testing institutions. It can be seen that the pattern distribution is consistent with the simulation results. In the range of $400-2000 \mathrm{MHz}$, the $-3 \mathrm{~dB}$ lobe width of the horizontal H-plane pattern is $360^{\circ}$, which shows that the biconical antenna meets the requirements of all items of detection. figure 8 shows the detection gain of biconical antenna at different frequencies. It can be seen that the gain is $-1.5 \sim 1.5 \mathrm{dBi}$ in the frequency range of 400 $2000 \mathrm{MHz}$, and the average value is about $0 \mathrm{dBi}$.
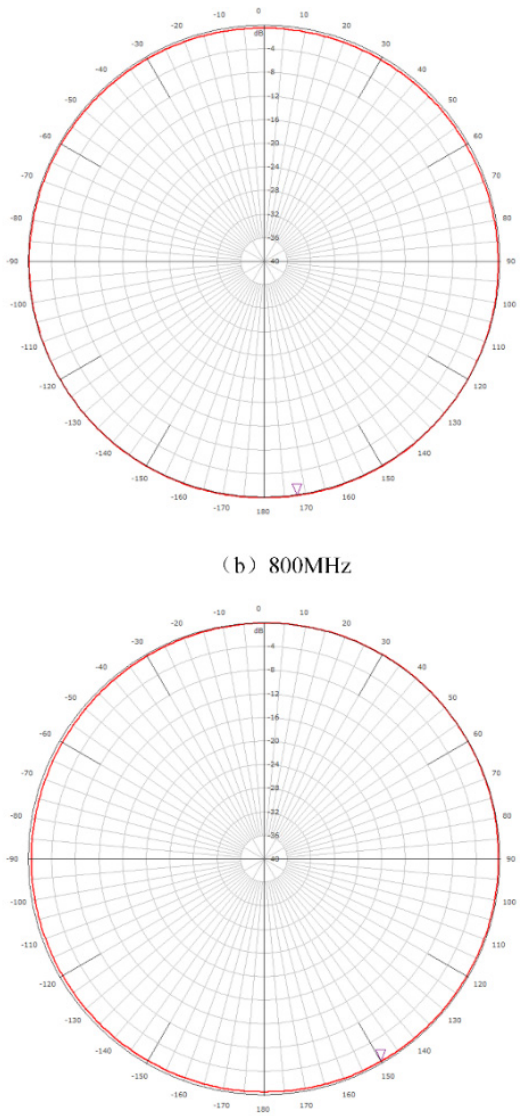

(d) $1600 \mathrm{MHz}$

Figure 7. Direction diagram of horizontal Plane H 


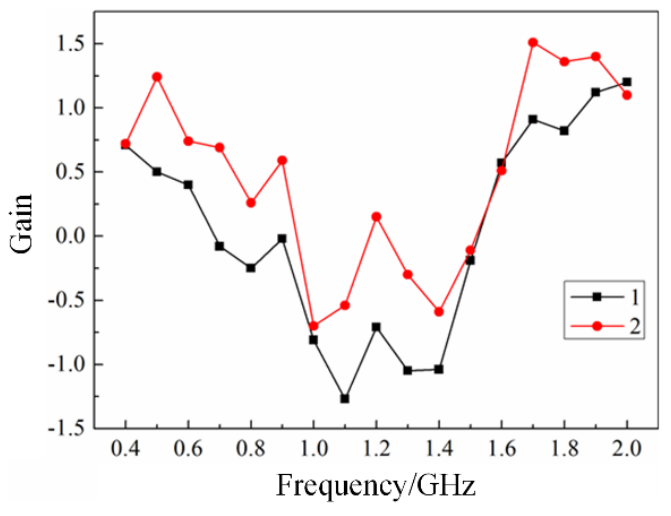

Figure 8. Gain curve of experimental biconical antenna

The equivalent height curve of the biconical antenna was tested in the GTEM chamber, and the test results are shown in figure 9. It can be seen that in the range of 0.1$2 \mathrm{GHz}$, the equivalent height of the biconical antenna is basically greater than $8 \mathrm{~mm}$, and the average equivalent height is about $13.3 \mathrm{~mm}$. At present, there is no general standard for equivalent height of UHF sensor in PD detection system of substation, but the state grid has equivalent height as GIS PD UHF sensor performance criterion, specified in the standard: GIS PD UHF sensor in $300 \mathrm{MHz}$ to $1500 \mathrm{MHz}$ bandwidth should be not less than $8 \mathrm{~mm}$, the average effective and least effective height should not be less than $3 \mathrm{~mm}$. Compared with this standard, the equivalent height of the bicone antenna in this system is much higher than $8 \mathrm{~mm}$. The test results also show that the bicone antenna has high sensitivity when receiving PD UHF signals.

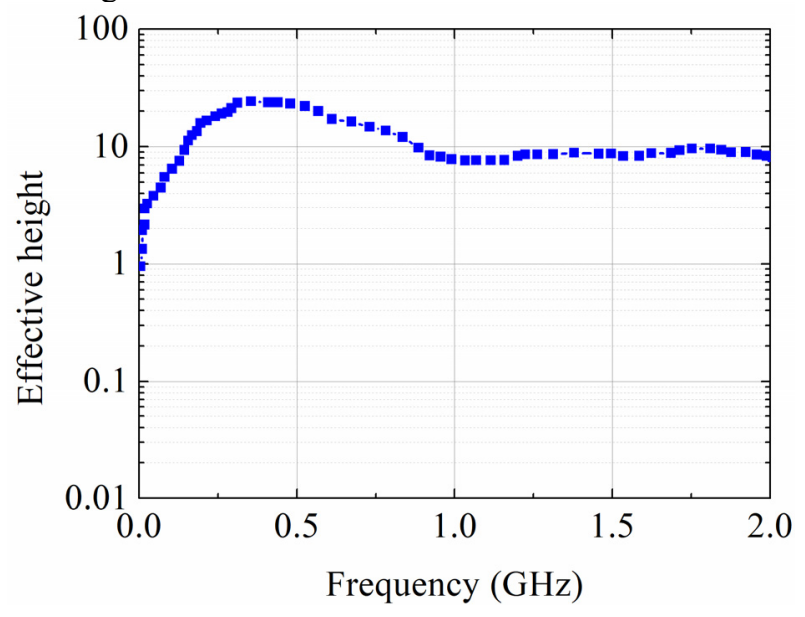

Figure 9. Equivalent height measurement results of the doublecone antenna

\section{Conclusion}

(1) According to the performance requirements of UHF antenna for PD detection in substations, the bicone antenna with better performance is selected as the UHF sensor. The influence of the structural parameters of the biconical antenna on the impedance bandwidth of the antenna is simulated. Aiming at the impedance bandwidth of the antenna within $0.3-3 \mathrm{GHz}$, the structure parameters of the antenna are optimized, and the antenna volume is not too large. A bicone antenna with good performance is designed.

(2) According to the design results, the bicone antenna is made, and the performance parameters such as the standing wave ratio and equivalent height of the antenna are tested. The test results show that the bicone antenna meets the requirements of $\mathrm{PD}$ detection and positioning of the substation, and the antenna has a good PD detection capability.

\section{Acknowledgements}

This work is supported by the Science and Technology Project of Shandong Electric Power Company (520609190003).

\section{References}

1. Zhang, M.Y., Li, L., Liu, Y.W., et al. (2020) Multiindices insulation condition assessment method for transformer based on grey matter-element method[J]. Journal of Electric Power Science and Technology, 35(1): 137-143.

2. Yuan, J.B., Xu, P.C., Li, L., et al. (2020) Prediction of transformer oil-paper insulation aging based on BP neural networks with the chicken swarm optimization algorithm[J]. Journal of Electric Power Science and Technology, 35(4): 33-41.

3. Zhou, J.S., Yu, J.F., Tang, S.F., et al. (2020) Research on temperature early warning system for substation equipments based on the mobile infrared temperature measurement $[\mathrm{J}]$. Journal of Electric Power Science and Technology, 35(1): 163-168.

4. Zhang, L.G., Lu, S.J., Li, C.R., et al. (2019) Observation on the Characteristics of Long-Term Partial Discharge Sparseness of Surface Metal Particle in Gas Insulated Switch[J]. Transactions of China Electrotechnical Society, 34(18): 3928-3936.

5. Liu, C.M. (2020) The comprehensive application of partial discharge online detection of gas insulated switchgear[J]. Electrical Engineering, 21(10): 117122.

6. Liu, Q., Chang, D.G., Deng, J.B. (2020) Optimal Selection on Spatial Spectrum Estimation Algorithms for UHF Direction Finding of Partial Discharge in Substation[J]. Transactions of China Electrotechnical Society, 35(16): 3551-3560.

7. Ma, S.J., Wang, P., Dong H., et al. (2020) Design of Ultra-high Frequency Antenna for Discharge Detection Under High Frequency Impulsive Voltage Conditions for Inverter-fed Motors Insulation[J]. High Voltage Engineering, 46(10): 3451-3459.

8. Ye, H.F., Qian, Y., Liu, Z.J., et al. (2015) A New UHF Sensor for Spatial Location of Partial Discharge Sources[J]. Transactions of China Electrotechnical Society, 30(8): 333-340.

9. Liu, X.Q., Zhou, Z.H., Cui, Y.J., et al. (2012) Development of discone antenna used for detecting corona of electrical equipments[J]. Advanced 
Technology of Electrical Engineering and Energy, 31(2): 74-78.

10. Ye, H.F., Qian, Y., Wang, J., et al. (2013) Research on UHF sensor for partial discharge spatial location in substation[J]. Power System Protection and Control, 41(19): 54-60.

11. Zhou, W.J., Liu, Y.S., Li, P.F., et al. (2017) Modified Vivaldi Antenna Applied to Detect Partial Discharge in Electrical Equipment Based on Ultra-High Frequency Method $[\mathrm{J}]$. Transactions of China Electrotechnical Society, 32(12): 259-267.

12. Sun, C.X., Xu, G.F., Tang, J., et al. (2004) Model and performance of inner sensors used for partial discharge detection in GIS[J]. Proceedings of the CSEE, 24(8): 89-94.

13. Hu, Y., Wang, H.B., Tang, L., et al. (2014) Research and Practice of Partial Discharge Online Monitoring and Locating for Substation Using UHF Sensor Array[J]. Transactions of China Electrotechnical Society. 29: 502-508.

14. Song, Z., Zhang, J.H., Huang, Z. (2011) Antennas and Radio wave Propagation[M]. Xidian University Press, Xian.

15. Zhong, S.S. (2011) Antenna Theory and Techniques[M]. Publishing House of Electronics Industry, Beijing.

16. Zhang, X.X., Chen, Y., Tang, J.Z., et al. (2011) Minitype Quasi-TEM Horn Antenna for Partial Discharge Detection in GIS[J]. High Voltage Engineering, 37(8): 1975-1981. 